\title{
Teleaudiencias: apuntes para la regulación de audiencias judiciales remotas
}

\section{Virtual hearings: Notes for the regulation of remote judicial hearings}

\author{
Renzo Joel Chaiña Durán ${ }^{[*]}$ \\ Erik Castellanos Tisoc ${ }^{[*]}$
}

\begin{abstract}
Resumen: el presente artículo constituye un estudio sobre las teleaudiencias. Tiene por objetivo explorar y analizar sus fundamentos y aplicación concreta, así como brindar los alcances necesarios para su eventual regulación abierta. Para alcanzar dicho objetivo, se acudió a la teoría aplicada al tema y se analizó su regulación nacional y extranjera. El artículo inicia desarrollando las bases de las teleaudiencias y su relevancia actual, luego de ello se hace una revisión de la normativa desarrollada en otros países, un análisis de su compatibilidad con los principios procesales y los posibles peligros de su aplicación, para inmediatamente después proponer tres modelos de regulación y finalmente hacer referencia a cómo se vienen desarrollado en el Perú.
\end{abstract}

Palabras claves: teleaudiencia, vistas telematicas, audiencias virtuales, videoconferencia.

Abstract: the present paper constitutes a study on virtual judicial hearings. Its objective is to explore and analyze its foundations and concrete application, as well as providing the necessary scope for its eventual open regulation. To achieve that objective, the theory applied to the subject has been revised and its national and foreign regulations were analyzed. The paper begins by developing the foundations of virtual judicial hearings and their current relevance, after which a review of the regulation developed in other countries is made, an analysis of their compatibility with procedural principles and the possible dangers of their application, for immediately then propose three regulatory models and finally refer to how they are developing in Peru.

Keywords: telehearing, telematic views, virtual hearing, videoconference.

[*] Bachiller en Derecho y Ciencias Políticas por la Universidad Nacional de San Antonio Abad del Cusco, miembro extraordinario del Círculo de Estudios Fraternidad Universitaria Cusco (CEFUC) - UNSAAC. Contacto: renzo_chad@outlook.com

[**] Bachiller en Derecho y Ciencias Políticas por la Universidad Nacional de San Antonio Abad del Cusco, miembro extraordinario del Centro de Investigación de los Estudiantes de Derecho (CIED) - UNSAAC. Contacto: castellanos.tisoc.erik@gmail.com 


\section{INTRODUCCIÓN}

A propósito del contexto pandémico que atraviesa el mundo, las relaciones sociales han sufrido un cambio en su dinámica. La prohibición de reuniones y, en general, las restricciones de movilidad han forzado al mundo a utilizar herramientas virtuales para su comunicación. Este fenómeno no ha sido ajeno al derecho, encontrando uno de sus principales tropiezos en la paralización de procesos judiciales. Siendo por ello necesario repensar la forma tradicional en que se llevan los procesos para que aterricen sobre las necesidades coyunturales, lo que como es evidente involucra a las audiencias judiciales y su naturaleza —por regla - presencial.

Ello supone enfocar nuestra atención a las Nuevas Tecnologías de Información y Comunicación (NTICs) que obtienen mayor relevancia en la administración de justicia, la implementación progresiva de la e-justice facilita la consolidación de una administración de justicia que tiene como ejes a la accesibilidad y la eficiencia. Su aprovechamiento no solo se sustancia en comunicaciones procesales y actos de mero trámite, sino también, en el propio desarrollo de un proceso llevado por audiencias, permitiendo que este sea remoto o a distancia, bajo la forma de teleaudiencias, como otro paso a dar hacia la desmaterialización del proceso.

Una teleaudiencia no exige la presencia física de -todos- los sujetos procesales en el mismo espacio; ya que, esta es reemplazada con el uso de tecnologías que permiten su interacción en tiempo real. Si bien las ventajas de su implementación serían notorias, pues - cuanto menos - estamos hablando de la optimización de principios procesales generales y específicos, el logro de un proceso judicial mucho más inclusivo, la simplificación de actos, evitar la suspensión innecesaria de sesiones de audiencia, la disminución de gastos; su irreflexiva aplicación también podría encarnar serias dificultades para el respeto de garantías procesales mínimas que aseguren la vigencia de los derechos de los justiciables, con especial incidencia en la inmediación, la prueba, la defensa y la igualdad.

Tales dificultades se trasuntan en una percepción negativa de la figura por parte de los órganos con capacidad normativa sobre la materia, los que establecieron una regulación tímida a las teleaudiencias, restringiéndolas a supuestos muy excepcionales. Lo que conllevó a que bajo el contexto de la COVID-19 se tenga impedimentos para llevar a cabo audiencias «no presenciales» y el consiguiente estancamiento masivo de procesos judiciales.

En tal sentido, el objeto de este aporte es revisar la figura de la teleaudiencia y explorar su naturaleza, condiciones y límites; todo ello con el fin de brindar luces para una reforma que posibilite el acercamiento a la ordinarización de las ellas; lo que, a su vez, permitirá suprimir, o cuando menos mitigar, barreras de índole temporal, espacial y económicas del proceso.

\section{CONCEPTO DE TELEAUDIENCIAS}

La teleaudiencia es una forma de llevar a cabo audiencias judiciales sin la presencia física de los sujetos procesales en el mismo espacio, para ello se acude a herramientas tecnológicas que, en tiempo real, permiten percibir el soma, expresiones físicas y verbales, en audio y video, y el contenido digital que compartan, logrando la interacción de todos los intervinientes. El término tele hace referencia a lo remoto entre uno y otro sujeto - espacialmente hablando-, mientras que audiencia, a la reunión que entre ellos se lleva a cabo para realizar los actos procesales que componen una audiencia judicial.

Para que se lleve a cabo, la audiencia requiere la presencia de determinados sujetos procesales. Esta presencia tradicionalmente fue entendida como exclusivamente física, dado que otrora no era posible llevar las audiencias con otra forma de presencia; sin embargo, a finales del siglo pasado los cuerpos legislativos $y$, principalmente, los tribunales empezaron a dar pase a las audiencias por videoconferencia 
asumiendo una nueva forma de presencia, la telepresencia (presencia virtual).

Es preciso aclarar lo que se entiende por videoconferencia y cuál es su diferencia con las teleaudiencias. Podemos entender a la videoconferencia como «(...) un servicio multimedia de comunicación que permite los encuentros a distancia en tiempo real entre distintos grupos de personas que se hallan en diferentes lugares» (Montesinos, 2009, p. 26). La premisa básica de la videoconferencia es permitir la comunicación multidireccional entre los sujetos de manera virtual. En síntesis, la teleaudiencia es la audiencia remota llevada con presencia virtual, mientras que la videoconferencia es la herramienta que lo hace posible.

\section{VENTAJAS DE LAS TELEAUDIENCIAS}

Existen múltiples antecedentes cuyas conclusiones precisan los beneficios que traen consigo las teleaudiencias.

De manera resumida se puede destacar, como refiere Fons (2008):

(...) la consecución de economía procesal, esto es, el ahorro de tiempo, dinero y trabajo, dado que se evitan gravosos (y costosos) desplazamientos, así como eventuales interrupciones o suspensiones de juicios, vistas y comparecencias (algunas podrían calificarse de dilaciones indebidas), toda vez que cabe lograr concentración y unidad en las actuaciones. (p. 54)

Como consecuencia, el proceso judicial será más célere, pues permitirá que todos los actos procesales se lleven con la menor cantidad de tiempo entre unos y otros.

Sobre la grabación de las videoconferencias afirma Amrani-Mekki (2008):

Evitan tener que convocar de nuevo a los testigos para escucharlos, ahorrándose de este modo el tiempo y coste de nuevas comparecencias. Por otra parte, la celeridad se encuentra íntimamente ligada a la calidad, ya que el paso del tiempo hace perder eficacia, por pérdida de frescura en la memoria, a los testimonios respecto de los hechos a los que se refieren. (p. 10)

Por su parte, sobre las teleaudiencias y la práctica de medios de prueba consistentes en la recepción de declaración a personas, en el reconocimiento de personas o en el reconocimiento de objetos que no puedan ser trasladados ante el tribunal Ortells (2002) postula:

Ciertamente estas técnicas también reducen los retrasos que se originan en la prestación del auxilio, pero su principal ventaja es la mejora de la calidad del acto de recepción de la prueba, porque posibilitan conservar, en gran medida, las ventajas que derivan de la inmediación para la mejor apreciación de la prueba. (p. 229)

Las teleaudiencias no solo contribuirían a reducir el número de juicios suspendidos sino también evitarían conflictos innecesarios al no tener la presencia física de las partes (Aba, 2009, p. 19), atenuando los peligros de intimidación y victimización.

El costo reducido y la rapidez de las teleaudiencias permiten garantizar de manera positiva el servicio de justicia, dado que el espacio ya no es una limitación. Por lo tanto, llega a ser una solución al requisito de una buena administración de justicia (Binet, 2005).

\section{DESARROLLO NORMATIVO DE LASTELEAU- DIENCIAS}

Conviene dar un repaso a la normativa establecida en otros ordenamientos para entender cómo las videoconferencias -y las teleaudiencias - han ido calando en la legislación y en la práctica jurídica.

Menciona Tirado (2017) que «(..) en un primer momento las videoconferencias fueron utilizadas incluso sin cobertura legal, (...) siendo el proceso penal el precursor en la utilización de este medio tecnológico, a finales de la última década del siglo pasado» (p. 55). 
En Bélgica y Japón, desde inicios de la década pasada, las videoconferencias se utilizan cuando una parte está fuera del tribunal (Jeuland, 2007).

En Inglaterra, respecto al proceso civil, destaca la Ley de Acceso a la Justicia de 1999, la cual permitió el uso de las videoconferencias «(..) cuando hay testigos que se encuentran en el extranjero, o en cualquier tipo de casos civiles en los cuales los tribunales lo autorizan o las partes involucradas en la causa consienten en su uso» (Lillo, 2011, p. 129).

En Francia, en 2007 con la promulgación de la Ley N. ${ }^{\circ}$ 2007-1787, Ley de Simplificación del Derecho, se dio un avance notable en pro de la modernización del proceso civil (Dimeglio, 2008) modificada el 2011 mediante Ley N. ${ }^{\circ}$ 2011-803, por medio de la cual se permitió que las audiencias puedan realizarse por medio de telecomunicaciones audiovisuales. Con tal fin se establecieron condiciones en torno al uso de videoconferencias para las audiencias, en el sentido de que se puede dar de oficio o solicitud de una de las partes pero siempre sometida a la decisión del juez, que la decisión judicial esté consentida por las partes del proceso, y que los debates de las audiencias sean abiertos al público. De similar modo, el artículo 128-a del Código Procesal Civil Alemán (Zivilprozessordnung, 1887), regula el procedimiento de transmisión de imagen y sonido a solicitud de parte, el cual permite que los actos procesales de las audiencias puedan ser transmitidos simultáneamente en el lugar donde las partes o sus representantes estuviesen.

En España se creó la Subdirección General de las Nuevas Tecnologías, entidad dependiente de la Dirección para la Modernización de la Administración de Justicia, que en dos etapas sucesivas, se encargó de la implantación del uso de la videoconferencia (Arnaiz, 2016, p. 8). A diferencia de otras legislaciones europeas, la española se caracteriza porque no necesita el consentimiento de una de las partes, los artículos 229.3 y 230.1 de la Ley
Orgánica del Poder Judicial (1985) establecen la posibilidad del uso de las videoconferencias por motivos de utilidad, seguridad y orden público, así como en aquellos supuestos en que la comparecencia resulte gravosa o perjudicial.

Sobre el uso de videoconferencias en el sistema anglosajón, 2017 Tirado (2017) apuntó:

El uso de la videoconferencia también se ha afianzado en los sistemas de tradición anglosajona (...), de modo que diversos países contemplan su empleo en el marco de su ordenamiento interno (Australia - Ley 1 de marzo de 1997-, Canadá — a través de la Ley de 17 de junio de 1999_, Estados Unidos —donde habiendo comenzado por las declaraciones de militares destinados en el extranjero, su práctica se extendió a menores víctimas y a testigos de determinados hechos delictivos-, Inglaterra, Irlanda, Islandia [...]). (p. 157)

En el plano regional, países como Argentina, Colombia, Paraguay, Venezuela y Brasil vienen realizando videoconferencias en materia penal, en más de alguno de ellos sin que exista norma específica que la prevea (Albornoz \& Magdic, 2013, pp. 249-251).

Dentro del derecho internacional se ha previsto el uso de las videoconferencias como herramienta de auxilio en procesos transfronterizos y a modo de cooperación para asuntos penales. De manera expresa el Estatuto de la Corte Penal Internacional (1998) en su artículo 69.2 permite al testigo presentar testimonio por medio de una grabación de vídeo o audio.

La Convención de Palermo sobre Delincuencia Organizada Transnacional de 2000, de las Naciones Unidas, permite en el numeral 18 del artículo 18 que la audiencia se celebre por videoconferencia si no es posible o conveniente que el sujeto comparezca personalmente en el territorio del Estado requirente. Similar es la regulación que se encuentra en la Convención de Naciones Unidas contra la Corrupción de Mérida. 
La Unión Europea, por su parte, aprobó en el año 2000 el Convenio Europeo Relativo a la Asistencia Judicial en Materia Penal que reguló la práctica de las videoconferencias de testigos, peritos y acusados cuando no sea oportuno o posible que la persona que se halla en el territorio de uno de sus Estados miembros comparezca en el territorio de otro Estado cuyas autoridades tengan necesidad de oírla.

Siguiendo esa línea, en 2013 el Consejo de la Unión Europea (Consilium) en su Guía sobre videoconferencias en procedimientos judiciales transfronterizos señaló:

El marco jurídico aplicable para la obtención y práctica de pruebas por videoconferencia en asuntos de Derecho civil y mercantil es el Reglamento (CE) N. ${ }^{\circ}$ 1206/2001 relativo a la cooperación entre los órganos jurisdiccionales de los Estados miembros en el ámbito de la obtención de pruebas en materia civil o mercantil. (p. 25)

El Tribunal Europeo de Derechos Humanos ha emitido pocas sentencias en esta área, pero tuvo la oportunidad, en varias ocasiones, de decidir sobre la compatibilidad de las videoconferencias con el derecho a un juicio justo (Laure, 2011).

Los países de América han suscrito varios instrumentos internacionales —tratados bilaterales y multilaterales - que regulan el uso de la videoconferencia. Destaca el Convenio Iberoamericano sobre el Uso de la Videoconferencia en la Cooperación Internacional entre Sistemas de Justicia, que tiene por objeto favorecer el uso de la videoconferencia entre las autoridades competentes de las partes como un medio concreto para fortalecer y agilizar la cooperación mutua en materia civil, comercial, penal y en otras materias que las partes acuerden. Siendo también relevante mencionar a los registros de la Reunión Especializada de Ministerios Públicos del Mercosur (REMPM) y específicamente su instrumento de trabajo denominado «Cuestionario de Salvador de Bahía sobre Videoconferencias», que estimó impor- tante la implementación de la videoconferencia en la cooperación internacional (Albornoz \& Magdic, 2013, p. 246).

\section{COMPATIBILIDAD CON LOS PRINCIPIOS PROCESALES}

Antes de reflexionar sobre la teleaudiencia y su compatibilidad con los principios procesales conviene definir la postura que se tomará en cuenta en adelante. En doctrina se puede identificar dos posturas bien marcadas. Por un lado, una que afirma que la implementación de las videoconferencias afectan per se principios procesales (Tirado, 2017, p. 167) lo que terminaría por limitar el uso de cualquier teleaudiencia - pues esta se encontraría próxima a la nulidad-; y, de otro lado, la que propugna que el empleo de las videoconferencias no afectan los principios procesales, pero que dicha compatibilidad estaría condicionada «(...) por la forma en que se acuerde su práctica o se lleve a cabo su ejecución, aseveración que es predicable respecto de la forma de práctica o ejecución de cualesquiera diligencias en las condiciones tradicionales sin empleo de medios tecnológicos» (Tirado, 2017, p. 168). Es importante efectuar tal diferencia puesto que de ella dependerá la forma de aplicación de las teleaudiencias: ya sea esta de modo excepcional - restringiendo su uso a determinados supuestos-; o, por el contrario, de modo generalizado - permitiendo su uso optativo-. El presente se suscribe a la segunda postura, precisando además que la vulneración de los principios quedaría supeditada no solo a la forma en que se practiquen las teleaudiencias sino también a la interpretación que se da a los principios como la disponibilidad a los recursos tecnológicos.

Demás está decir que la administración pública no puede quedarse al margen del desarrollo y la implementación de las NTIC; sin embargo, la eficacia por eficacia puede traer consigo a la vulneración de principios que terminarían por nulificar el acto de audiencia. Más 
adelante se podrá advertir que los cuestionamientos sobre la vulneración de principios se sustentan en un corto alcance de estos y que además no se cuenta con la tecnología adecuada para que una audiencia virtual tenga la misma fluidez y desarrollo que una presencial. Argumentos que van perdiendo validez frente a un derecho que día a día ajusta sus instituciones a una sociedad cuya modernización tecnológica crece a pasos agigantados, dejando así abierta la posibilidad de que en un futuro próximo se tengan por solucionadas las cuestiones de interpretación y la escasez de recursos tecnológicos.

Su regulación, por tanto, debe ser elaborada cuidadosamente, en paralelo al respeto del ordenamiento jurídico. Su uso no se debe sostener simplemente en el avance tecnológico y el provecho que se pueda sacar, se debe dar al servicio de la administración de justicia, pues de ser lo primero, se ampliaría la posibilidad de caer en indebidas prácticas judiciales, exponiendo el proceso incluso a más vicios de los que ya existen. Las teleaudiencias no se pueden ejecutar mutilando los objetivos de la administración de justicia ni sobrepasando los límites institucionales del órgano jurisdiccional, por lo que, es imprescindible realizar un adecuado encaje de las teleaudiencias al ordenamiento jurídico para así estas no vulneren principios, únicamente bajo esa premisa es que la teleaudiencia podrá encontrar una real y eficiente aplicación.

\subsection{Principio de publicidad}

Varios estudios han demostrado que el uso de las videoconferencias no tiene incidencia en el principio de publicidad.

\section{Sostiene Amoni (2014):}

[Si] (...) la audiencia virtual se realiza en una sala de audiencia a la que puede ingresar quien lo deseare según la capacidad del recinto y el tipo de proceso que se esté llevando a cabo, al proyectarse en una pantalla adecuada al espacio y reproducirse con un sistema de sonido con capacidad apta para el mismo lugar en que se presenta la declaración o debate telemático, además de una velocidad de conexión de Internet suficiente, se cumpliría con el referido principio de publicidad, al igual que si el sujeto procesal estuviese presente. (p. 15)

De similar modo concluye Carrizo (2008) al afirmar que «(...) en relación con la posible afectación del principio de publicidad, entendemos que el recurso a la videoconferencia posibilita las mismas condiciones de publicidad que podrían darse en la realización de cualquier acto procesal». (p. 145)

Por otro lado, verificada la compatibilidad, conviene repasar sobre las bases del principio de publicidad para explicar cómo las teleaudiencias le favorecen.

La fundamentación de este principio, como sostiene Monroy (1996):

[E]s que el servicio de justicia es un servicio social. Esto significa que lo que ocurre en los tribunales no es de interés exclusivo de los litigantes sino de la sociedad. Qué se hace para que se resuelvan los conflictos es una información que debe ser conocida por la comunidad. Con tales datos, podrá establecerse una relación de confianza entre los órganos jurisdiccionales y su comunidad. (p. 82)

En suma, si la publicidad implica la proscripción de clandestinidad de la audiencia -permitiendo sobre ella participación y control- y el aseguramiento del mayor acceso posible a esta, las teleaudiencias serían un mecanismo idóneo para salvaguardarla. Entendiendo además que, al obrar en soporte digital, este permitiría el acceso a la información para un mayor número de personas que podrían conectarse electrónicamente desde cualquier lugar. Lo que no sucede con las audiencias físicas, en las que los terceros deben concurrir necesariamente a la sala de audiencias, salvo aquellas escasas audiencias que hayan sido 
transmitidas en directo (como solo ocurre con los casos mediáticos) y aquellas que hayan sido grabadas y publicadas, último supuesto en que el acceso será posterior a la realización de la audiencia y no en tiempo real, como sí sucede con las teleaudiencias.

Bajo lo explicado en este apartado, las teleaudiencias no solo cumplen condiciones mínimas de publicidad, sino que las mejoran, pues admiten la posibilidad de «asistencia» de más personas —crece el auditorio-y permite un mejor seguimiento a los procesos judiciales. En otras palabras, al no limitarse a una presencia física, personas que se encuentran en otras latitudes pueden tener acceso, bastando con que se publique la hora y fecha de la audiencia con el enlace para observarla.

\subsection{Principio de oralidad}

Es evidente pensar que «(...) debido a la propia naturaleza de la videoconferencia como instrumento que permite la comunicación bidireccional de la imagen y el sonido, esta quedaría suficientemente garantizada». (Carrizo, 2008, p. 146)

Incorporando las NTIC a las audiencias, se garantiza este principio, ya que las partes, testigos, peritos e intérpretes pueden comunicarse oralmente con el juzgador y viceversa, e inclusive entre ellos (Amoni, 2014, p. 14); por lo que, «(...) tampoco ofrece problemas acatar el principio de oralidad, de hecho, ésta es consustancial a la videoconferencia en cuanto instrumento de comunicación oral y visual. Pocas palabras hay que dedicar para justificar lo evidente». (Tirado, 2017, p. 169)

\subsection{Principio de contradicción}

Este principio apunta Peláez (2015):

Implica la necesidad de una dualidad de partes que sostienen posiciones jurídicas opuestas entre sí, de manera que el tribunal encargado de instruir el caso y dictar sentencia no ocupa ninguna postura en el liti- gio, limitándose a juzgar de manera imparcial acorde a las pretensiones y alegaciones de las partes. (p. 29)

Carrizo (2008) refiere sobre el tema en el proceso penal:

Tampoco consideramos que la contradicción pueda verse comprometida por cuanto los defensores de las partes podrían efectuar las preguntas que sean declaradas pertinentes, tanto a los testigos o peritos que pudieran declarar por el sistema de videoconferencia, como a los acusados que se encuentren en algún centro penitenciario, dando fe el secretario judicial que se encuentre junto al testigo, perito o acusado, de la correcta y concreta recepción de las preguntas que formule tanto el tribunal, como el Ministerio Fiscal y el resto de las partes presentes en el acto, así como de las contestaciones que se den a las preguntas formuladas y que serán cotejadas con la presencia, al mismo tiempo, del secretario judicial que se encuentra físicamente en la Sala. (p. 146)

La misma lógica — con algunos ajustespuede emplearse con el resto de procesos judiciales, toda vez que mientras se garantice la bidireccionalidad y la comunicación fluida en tiempo real, este principio no se verá afectado. La videoconferencia no restringe de modo alguno la posibilidad que tienen los defensores de formular los recursos, observaciones y precisiones en audiencia.

\subsection{Principio de concentración}

Principio en cuya virtud se exige que los actos procesales sean desarrollados de manera próxima unos a otros. Las audiencias pueden llevarse a cabo por tiempos más prolongados, es posible llevar a cabo mayor cantidad de actos procesales (Lillo, 2011, p. 129), en una sola sesión, o reducir los periodos en su intermitencia. Se colige entonces, que el principio de concentración no se afecta con la implementación de las teleaudiencias, por el contrario se garantiza y optimiza. 


\subsection{Principio de inmediación}

Principio que se encuentra orientado a que el juzgador entre en contacto directo con los elementos del proceso, logre acopiar los mayores detalles posibles y con ello emita un juicio sobre la base de lo percibido. Bien apunta Gimeno (s.f.), citado por Baral y Reissier (2014), que «solo el que ha sido testigo de todo el procedimiento, escuchó los argumentos de las partes y presenció la actuación de las pruebas, está legitimado para emitir sentencia». (p. 9)

Echandia (1993), citado por Amoni (2013), da a conocer que:

El principio de inmediación está constituido por tres aspectos: la proximidad entre el juez y lo que evaluará o a quienes evaluará; la inexistencia de intermediarios, bien fueren cosas o personas, y la bilateralidad, de donde derivan dos tipos de inmediación: la pasiva, que supone la posibilidad del juzgador de percibir directamente la pruebas, (...); y la activa, que consiste en la percepción e intervención directa en el conocimiento de las pruebas por parte del juzgador (...). (p. 74)

Por lo tanto, el uso adecuado de la videoconferencia deberá respetar todos los aspectos que importan al principio de inmediación, es decir que se busque que las partes del proceso tengan la mayor visibilidad entre ellos, que no exista intermediación entre los sujetos que por su condición deban actuar directamente en el proceso, y que se pueda visualizar las pruebas con la posibilidad de intervenir sobre ellas.

Como es evidente, las ideas de inmediación y presencia jurídica son inescindibles, por lo que es el principio de inmediación en el que más incide las teleaudiencias. En tal sentido, es de suma relevancia la interpretación que se realice de este principio a efectos de que tenga consonancia con la realidad y las necesidades de una sociedad como la nuestra.

Una concepción tradicional de este principio concluiría, según Tayro (2016) que «el principio de inmediación se cristaliza solo en la presencia física y la interrelación directa y frontal entre [los sujetos procesales]; de forma que permita conocer al juez, no solo a la persona, sus declaraciones, sino su personalidad, actitud, reacciones» (p. 553). Concepción que nos lleva a dos conclusiones: Que, en supuestos de aplicación ordinaria, las teleaudiencias no son viables dado que no satisfacen la presencia física exigida por el principio de inmediación; y, que, en supuestos estrictamente excepcionales, el principio de inmediación debe ser «sacrificado» para realizar las teleaudiencias - en caso existan principios distintos con un peso concreto superior-.

Otra interpretación de este principio determina alcances mayores, pues como refiere Amoni (2013) sobre la relación existente entre los avances tecnológicos y el principio de inmediación procesal «(...) esta tecnología constituye un medio para acercar en tiempo real a personas alejadas geográficamente y así permitir su interacción audiovisual, que es en definitiva lo que inspira al principio de inmediación» (p. 74). Lectura del principio bajo la cual puede verse garantizado por las teleaudiencias, siendo posible no solo su aplicación, sino su exigibilidad.

Para definir entre uno u otro de estos sentidos, se debe tener en cuenta que «(...) el respeto de la adecuada relación que debe existir entre el órgano jurisdiccional y lo que constituye el objeto del proceso, solo podría verse salvado desde la aceptación de una presencia virtual que pudiera ser equivalente a la presencia física» (Carrizo, s.f). Es decir, podrá aceptarse la presencia virtual en tanto satisfaga los estándares mínimos del principio de inmediación y las reglas que este haya inspirado bajo la forma de presencia física.

Se tiene, primero, que el contacto directo con los elementos subjetivos del proceso al que se alude cuando hablamos del principio de inmediación implica «(...) que el juez y quien declara estén ubicados cerca del otro para que la declaración pueda ser percibida por el 
administrador de justicia directamente y (...) sin intermediarios, de manera que el tribunal pueda formarse una decisión de la observación y escucha (...) de 'primera mano' y no tergiversada por la representación que pudieran efectuar terceras personas o cosas» (Amoni, 2013, p. 74). Segundo, que el contacto con los elementos objetivos, requiere un menor nivel de intensidad de inmediación, dado que no está supeditado a la percepción de expresiones físicas o verbales. Entonces, se puede inferir que el contacto directo puede ser desligado de la idea de cercanía en el plano espacial, ya que la concurrencia del sujeto y la percepción que tenga el juzgador de este y sus expresiones en las teleaudiencias - y viceversa - se manifiesta también de manera remota.

Así mismo, si en las audiencias físicas no deben existir intermediarios, esto tampoco es posible en las teleaudiencias, recibiendo los sujetos y elementos de prueba igual cobertura. Ocurriendo algo similar respecto a la inmediación pasiva y activa, pues la comunicación con el representante del órgano jurisdiccional es bidireccional, y por ende este no solo percibe lo que ocurre en la audiencia sino que participa activamente en ella interactuando con los elementos del proceso.

De todo lo señalado se concluye que la presencia virtual que permite la teleaudiencia es equiparable a la presencia física, encontrando iguales niveles de satisfacción del principio. Afirmación última que hace necesaria la reescritura de la noción de presencia-física - hacia una presencia jurídica —-física y virtual-, para pasar de una concepción antitética entre teleaudiencias y principio de inmediación a una de coherencia, e incluso de optimización.

Un ejemplo las teleaudiencias como medida de optimización del principio de inmediación se puede graficar claramente en el caso de la declaración por exhorto en la que «(...) la videoconferencia refuerza el principio de inmediación, ya que la práctica de la prueba tiene lugar directamente ante el órgano que debe conocer el caso y no ante el tribunal que tuvo que practicar la asistencia legal» (Baral \& Reissier, 2014, p. 13).

Agregando a lo anterior, una de las principales críticas que reciben las teleaudiencias cuando contienen actuación probatoria se encuentra sin lugar a dudas sobre las declaraciones de testigos y peritos, es decir sobre la implicancia de la virtualización de las audiencias en las denominadas pruebas orales. Ello sin tomar en cuenta los aportes de la psicología del testimonio y el paso del modelo subjetivo de razonamiento probatorio —en el que el juez es el centro de la prueba - hacía un modelo objetivo - con un juez que no es el centro de la prueba-, en el que el contacto directo entre juez y declarante no es determinante para que el primero determine la veracidad de lo dicho por el segundo (de Paula Ramos, 2019. pp. 125-155). Tampoco es de acogida sostener que las expresiones corporales pueden ser tomadas como indicios de falsedad de lo declarado, pues ello no solo desbarata la crítica a la teleaudiencia, sino que implica alejar las conclusiones del órgano jurisdiccional de parámetros epistémicos y por ende privarlas de rigor científico.

\section{SOPORTE TÉCNICO Y PERSONAL ENCAR- GADO}

Aunque exista normativa ampliamente permisiva para las teleaudiencias, su aplicación no será viable en tanto no se cuente con el equipamiento necesario para su ejecución ni se haya capacitado al personal involucrado en la administración de justicia. Las reglas son insuficientes para dotarlas de un soporte real. Como se mencionó, la mejor tutela de principios involucra la adecuada implementación y utilización de herramientas tecnológicas en el proceso.

Las teleaudiencias presuponen la implementación de tecnologías que provean una conexión que permita una comunicación parecida a la real, fluida y sin interrupciones, pues 
«(..) es indispensable que los equipos que conforman los recursos audiovisuales permitan que el juez y los demás sujetos procesales se observen y escuchen con detalle, al mismo momento en que se producen sus manifestaciones, como si estuvieran uno frente al otro» (Amoni, 2013, p. 75). Lo mencionado impone al Estado la responsabilidad de «(...) realizar los estudios de factibilidad técnica y, en definitiva, dotar de estas herramientas tecnológicas a los diversos juzgados (...) y así poder llevar a cabo la correcta ejecución de la justicia (...)». (Retamales, 2017, p. 76)

Refieren Baral \& Reissier (2014), que:

El uso de la videoconferencia (...) impone muchas obligaciones a los estados: la obligación de proporcionar los medios necesarios, la obligación de estar alerta tanto para prevenir incidentes técnicos como para garantizar el respeto de los derechos de la defensa (...), [y] la formación de actores de la justicia en estas técnicas de comunicación.

La incorporación de NTIC pone de manifiesto el imprescindible papel que ocupan las autoridades en el manejo del presupuesto para la adquisición de herramientas y la formación de los servidores de la administración de justicia en su empleo. Sobre el particular es paradigmático el caso Lawyer Partner SA vs Slovenia, en el que el Tribunal Europeo condenó el hecho que la legislación eslovena preveía la posibilidad de presentar peticiones electrónicamente, pero que los tribunales se hayan negado a tramitar tales peticiones en ausencia de equipo técnico suficiente (Laure, 2011).

En resumen, la implementación de las teleaudiencias no encuentra problemas en lo dogmático, siendo la preocupación eminentemente de aplicación técnica, ya que este último tiene un impacto en los objetivos del proceso, en las garantías a salvaguardar y por tanto en los derechos de las partes. Es así que la atención debe enfocarse en los aspectos técnicos, los cuales de ninguna manera deben obstaculizar la comunicación entre las partes, encon- trandose los Estados obligados a asegurar la mejor calidad posible en las interacciones.

\section{MODELOS DE REGULACIÓN PARA LAS TELEAUDIENCIAS}

Revisada la literatura académica y la regulación sobre teleaudiencia en diferentes países, se puede sintetizar su uso en tres modelos bajo criterios de habilitación. Los cuáles serán expuestos precisando la naturaleza y alcance de cada uno, y las figuras jurídicas en que se desarrollan. Dependerá del sistema jurídico y de las tecnologías disponibles para optar por alguno de ellos.

\subsection{Modelo Cerrado}

Un modelo cerrado de regulación es aquel que establece una lista numerus clausus de supuestos de aplicación de teleaudiencias por razones de necesidad, con el fin de dar protección a algún interés tutelable o evitar un eventual peligro. Siendo por ello esta lista muy corta y con carácter estrictamente excepcional subordinado principalmente a consideraciones legislativas.

Aún cuando se hace referencia a un modelo cerrado, en la actualidad es inviable la absoluta proscripción de las teleaudiencias, incluso cuando se acepten como válidas las críticas que se le formulen. La vida moderna obliga a las autoridades a prever su uso, aun cuando tal posibilidad se reduzca a escasos supuestos.

Se está frente a circunstancias que exigen medidas dirigidas a salvaguardar derechos de los sujetos procesales o intereses nacionales -no para optimizar principios, sino para evitar su vulneración-. Por lo que, bajo este modelo se prevén teleaudiencias para sujetos en condiciones especiales de vulnerabilidad y otros supuestos como la declaración de personas que se encuentran en el extranjero o la actuación de personas con impedimentos materiales y/o legales para concurrir a la audiencia - por ejemplo personas con impedimento de salida del país o de una localidad-. 
Es ineludible reflexionar sobre el Proyecto de Reforma del Código Procesal Civil peruano (2018) —norma que en mayor o menor medida tendrá injerencia en el resto de proceso judiciales-. El proyecto señala en su exposición de motivos que son ejes de la reforma procesal civil el antiformalismo y la materialización de la e-justice a nivel transaccional. Proponiendo la eliminación de «repudiadas» formalidades innecesarias y la previsión de actuaciones procesales a través de medios técnicos y virtuales. Lo anterior resulta contradictorio cuando solamente abre las posibilidades de utilizar videoconferencias a personas en estado de vulnerabilidad y siempre que sea necesario (Art. 127- $\mathrm{A}^{\circ}$ - Participación en procesos judiciales de personas en condición de vulnerabilidad). Como es lógico, la intersección entre casos necesarios y casos con personas en situación de vulnerabilidad resulta en una regulación muy restrictiva.

\subsection{Modelo semiabierto}

Tomando como referencia la norma española (Arnaiz, 2016, p. 18), un modelo semiabierto es aquel que establece una lista numerus apertus de aplicación de teleaudiencias, que amplía los supuestos por razones de oportunidad, que suelen ser sometidos al criterio del órgano jurisdiccional. Es decir, toman en consideración supuestos que escapan de lo estrictamente necesario, pero sin llegar a considerar su aplicación indiscriminada. Para casos en los que resulte conveniente en atención al tiempo y lugar, evitando una comparecencia dificultosa o gravosa.

Con este modelo se aprovechan los beneficios de la teleaudiencia, ya que no se circunscribe a excepcionalidades sino a su funcionalidad como medio de ahorro de recursos económicos y de tiempo, bajo consideraciones legislativas y jurisdiccionales.

Bajo este modelo se asume una predisposición por las teleaudiencias que se ve limitada, por un lado, por razones de acceso tecnológico, infraestructura y presupuesto, y, por el otro, por la posibilidad de vulnerar derechos o servir para llevar a cabo actos maliciosos. En supuestos no legislados, corresponderá a los jueces evaluar cada caso y resolver si corresponde -o no- realizar una teleaudiencia. Por tanto, para el modelo semiabierto «(...) debe existir un control de equilibrio entre los derechos de las partes y la efectividad del proceso, que constituiría el principal razonamiento» (Laure, 2011) del magistrado para poder determinar la procedibilidad de las teleaudiencias.

Es fundamental tomar en cuenta todo lo anterior para establecer de manera adecuada el uso de las teleaudiencias y controlar su ejecución. Son los jueces los encargados de garantizar que las teleaudiencias se limiten a los supuestos en los que amerite su aplicación y que durante todo el desarrollo de las videoconferencias se proporcionen los medios necesarios y se respeten todas las garantías.

Es así que el test de proporcionalidad se presenta como una herramienta en la que el juez se puede valer para certificar el uso de las teleaudiencias. Sobre este tema, Tirado (2017) comenta que:

Deben concurrir los tradicionales requisitos de necesidad, adecuación y proporcionalidad exigibles conforme a la doctrina general de restricción en materia de derechos fundamentales. Por el requisito de la necesidad, es preciso tener en cuenta y fundamentar cómo no existen otras medidas adoptables que con igual o parecida eficacia puedan conducir a los mismos resultados. Por el presupuesto de la idoneidad resulta necesario considerar y motivar suficientemente cómo el uso de este avance tecnológico es adecuado para la consecución del fin legítimo perseguido, conduciendo naturalmente al mismo. Por la exigencia de proporcionalidad hay que plantearse la aptitud de la videoconferencia para la obtención del fin perseguido en cada supuesto, contemplando la relación razonable entre el resultado buscado y las renuncias a las condiciones 
normales de celebración del acto que resultan necesarias para lograr ese objetivo. Hay que valorar ya desde el inicio la gravedad del hecho o de la situación y su suficiencia para justificar la aplicación de este recurso técnico. En definitiva, su práctica debe estar suficiente y expresamente motivada en razones de este tenor. (p. 171)

Sería útil en el caso de que la distancia fuera tal que el coste de desplazamiento resulte desproporcionado (Amrani-Mekki, 2008). Un claro ejemplo de aplicación de este modelo son las audiencias de segunda instancia y de casación. En las que se permite teleaudiencias dada la naturaleza del debate, evitando que tengan que viajar hasta los tribunales para ahorrar tiempo, recursos en transporte y viáticos que pueden ser invertidos en una mejor defensa.

\subsection{Modelo abierto}

Un modelo abierto es aquel que no tiene una lista de supuestos de aplicación, pues esta obedece a razones de practicidad. Modelo que se basa en la voluntad de los sujetos procesales y se dará una vez superada las barreras tecnológicas y la teleaudiencia tenga la misma categoría que una audiencia física.

Formas de aplicación de este modelo pueden ocurrir por convalidación o convencionalidad. Se habla de convencionalidad cuando, bajo acuerdo, las partes soliciten acudir al proceso por videoconferencia; y, de convalidación, cuando habiéndose programado la teleaudiencia de oficio o a solicitud de solo una de las partes, no se formulan observaciones a su instalación. Supuestos en los que se realiza la teleaudiencia con conformidad del juez y las partes. Ante la solicitud deberá anoticiar al resto de sujetos procesales - que pudieran verse afectados-, quienes podrán oponerse.

El modelo abierto tiende a la masificación de las teleaudiencias extendiendo su aplicación a todas las fases de cualquier proceso judicial. No obstante, este no tiene por efecto desnaturalizar actos que por su esencia son presenciales, como las audiencias civiles de inspección judicial.

\subsection{Principio de Fidelidad a la realidad}

Por el principio de fidelidad a la realidad «(...) debe intentarse que la sesión de videoconferencia guarde el máximo parecido posible con la práctica habitual de cualquier órgano jurisdiccional» (Consilium, 2013, p. 17). Por lo que, la realización de la teleaudiencia debe ser lo más parecida a una audiencia física, debe existir identidad entre ambas, obligando a seguir los mismos procedimientos y formalidades.

Es preciso resaltar que este principio principalmente funcionaria en el modelo cerrado y semiabierto, ya que en estos las teleaudiencias se encuentran supeditadas a la audiencia física. Sin embargo, es casi seguro que las teleaudiencias dejen de estar limitadas a su uso y terminen imponiéndose en un futuro, dando lugar al diseño de una nueva forma de audiencia alternativa a la física, que variaría actos - como la acreditación de los sujetos procesales-. Se anota que, si bien actualmente las teleaudiencias merecen una regulación que atienda a cubrir las características que pueda soportar cada sistema jurídico, en un futuro no resulta irrazonable la idea de otorgar un procedimiento de audiencia distinto al de la audiencia física.

\section{TELEAUDIENCIAS EN EL PERÚ}

\subsection{Antecedentes}

La primera vez que se realizó una audiencia remota en Perú fue en diciembre del 2006 para una demanda de adopción en el Expediente $\mathrm{N} .^{\circ} 2311-2005$, siendo un antecedente importante para las teleaudiencias en materia de familia. (Torres, 2009, p. 158)

No obstante, las teleaudiencias tomaron mayor relevancia en los procesos penales por razones de seguridad y orden público, así como para disminuir el peligro procesal. Razón de ello es que en el año 2010 se creó el Sistema Nacional de Videoconferencias del Poder 
Judicial para optimizar la forma en que se llevaban a cabo las teleaudiencias en procesos penales, sistema que se vio reforzado con la dación de las siguientes directivas:

i. Directiva 01-2013-CE-PJ, «Procedimiento para la Ejecución de Audiencias Virtuales»;

ii. Directiva 01-2014-CE-PJ, «Lineamientos Para el Uso de la Videoconferencia en los Procesos Penales», con la que se permitió la participación de víctimas, testigos y peritos cuando su presencia física sea imposible, difícil o muy gravosa; $y$,

iii. Directiva N. ${ }^{\circ}$ 005-2015-CE.-PJ, «Procedimiento Para el Uso de Equipos de Videoconferencia por Personal de los Órganos Jurisdiccionales y Administrativos del Poder Judicial Peruano», que tiene por objetivo garantizar una adecuada utilización de los equipos del Sistema Nacional de Videoconferencias del Poder Judicial.

Así mismo, en el año 2013 se incorporó el artículo 119-A.2 al Código Procesal Penal (2004) peruano mediante la Ley $N{ }^{\circ} 30076$, que ad litteram establece que «(...) excepcionalmente, a pedido del fiscal, del imputado o por disposición del juez, podrá utilizarse el método de videoconferencia en casos que el imputado se encuentre privado de su libertad y su traslado al lugar de la audiencia encuentre dificultades por la distancia o porque exista peligro de fuga».

En el campo jurisprudencial, el Tribunal Constitucional peruano afirmó que las audiencias por videoconferencias no impedían que los sujetos procesales se comuniquen oralmente, dado que la interacción y diálogo se mantenía con este «sistema», siempre y cuando se garanticen condiciones técnicas mínimas que no obsten la percepción sensorial. Pese a todo lo dicho, este colegiado sostuvo que el uso de la videoconferencia no debe ser la regla general, sino una medida de empleo excepcional (STC Exp. N. ${ }^{\circ}$ 02738-2014-PHC, 2015), pronunciándose en el mismo sentido la Corte Suprema peruana. (CSJ R.N. N. ${ }^{\circ}$ 999-2016-Loreto, 2017)

En consecuencia, la videoconferencia en el Perú —en materia procesal penal— no solo tiene un marco jurídico que la sustenta, sino que fue sometida a control constitucional por el máximo intérprete de la Constitución, que validó la norma y su aplicación. (Tayro, 2016)

\subsection{Actualidad (Aislamiento social)}

El Poder Judicial no se encontraba preparado cuando se suscitó la emergencia sanitaria a consecuencia de la propagación de la COVID-19, declarada mediante el D. S. N. ${ }^{\circ}$ 008-2020-SA ${ }^{[1]}$, y se decretó el régimen de excepción mediante D. S. N. ${ }^{\circ}$ 044-2020-PCM ${ }^{[2]}$ con la restricción del ejercicio de los derechos constitucionales de libertad de tránsito y libertad de reunión pacífica libre de armas.

Lo anterior obligó a que se tomen medidas improvisadas como la Resolución Administrativa 103-2020-CE-PJ del Consejo Ejecutivo del Poder Judicial (CEPJ) que implementa el «Plan de prevención del coronavirus (COVID-19) en el Poder Judicial» estableciendo el servicio de administración de justicia mediante el teletrabajo de magistrados y auxiliares jurisdiccionales. Resolución que motivó a cada Corte Superior de Justicia del país a regular su funcionamiento interno y decidir los casos en los que tenía que continuar el trabajo, molecularizando la regulación a nivel de 35 Cortes Superiores.

El aislamiento social obligatorio pudo ser entendido como un supuesto perfectamente subsumible en la regulación actual sobre

[1] «Decreto Supremo que declara en Emergencia Sanitaria a nivel nacional por el plazo de noventa (90) días calendario y dicta medidas de prevención y control del COVID-19", de fecha 11 de marzo de 2020

[2] «Decreto Supremo que declara Estado de Emergencia Nacional por las graves circunstancias que afectan la vida de la Nación a consecuencia del brote del COVID-19», del 15 de marzo de 2020 
teleaudiencias. A pesar de ello, solo se acude a las audiencias judiciales por videoconferencia para «servicios esenciales» (procesos de garantías de libertad -en los que existan presos en situación de inocencia y condenados con ejecución efectiva de penas privativas de libertad- $y$ procesos urgentes - como los de materia constitucional y de familia-). Los demás procesos quedaron detenidos, disponiéndose la suspensión de plazos procesales (Arellano, Cora, García, \& Sucunza, 2020 pp. 70-71). Posteriormente, los auxiliares jurisdiccionales tuvieron que programar las audiencias «urgentes", pues no realizar dichas audiencias hubiera conllevado consecuencias nocivas.

Entre las herramientas que emplearon se encuentra a la aplicación «Google Hangouts Meet», que mediante Acuerdo 482-2020[3] del CEPJ fue declarada como herramienta para realizar teleaudiencias y reuniones administrativas -en tanto permanezca la emergencia sanitaria declarada en el país-. También se permitió el uso de la plataforma para las comunicaciones entre abogados y litigantes con los jueces y administradores de los módulos de justicia del país mediante Resolución Administrativa N. ${ }^{\circ}$ 000123-2020-CE-PJ del CEPJ, del 11 de marzo del 2020.

La práctica virtual de las audiencias improrrogables terminó por evidenciar que las brechas tecnológicas para las teleaudiencias no tienen la dimensión que se les asigna y que incluso en un contexto de emergencia las medidas fueron implementadas, llegando a ser reconocidas desde la sociedad, pues se informó de la realización satisfactoria de más de 9917 teleaudiencias hasta el mes de mayo (Marquina, 2020). Concluyendo que desde luego existen brechas tecnológicas en lo que se refiere al acceso y uso de las NTIC, pero estas pueden ser superadas.

Una de las medidas implementadas para disminuir la brecha tecnológica fue la capacita- ción para el uso de la plataforma mencionada por parte de las Cortes Superiores y Colegios Profesionales, para tal fin se convocó de manera segmentada a litigantes, personal administrativo y magistrados ; así mismo, en fecha 25 de junio de 2020 mediante Resolución Administrativa N. ${ }^{\circ} 000173-2020-C E-P J$ que aprueba el «Protocolo Temporal para Audiencias Judiciales Durante el Periodo de Emergencia Sanitaria" se estableció que el auxiliar jurisidiccional de juzgado haga lo necesario para el éxito de cada audiencia mediante la denominada reunión de actos preparatorios. Ello evidenció que no se requería de un presupuesto elevado para capacitación e implementación. Tampoco se necesitó contar con presupuesto ni personal informático distinto al que ya se tenía contando, sumado a que las Cortes Superiores mantuvieron sus propios sistemas de comunicación telefónica con los usuarios.

Otra medida fue la tomada por el Consejo Ejecutivo del Poder Judicial y la Corte Superior de Justicia de Lima, que instaron a que se realice trabajo remoto de los órganos en los que se haya implementado el expediente judicial electrónico (EJE). Medida que es aplicable a escasos órganos jurisdiccionales en el país, ya que el EJE se encuentra en contadas cortes del país - principalmente en la ciudad de Lima-, entre los cuales no se encuentran todas las especialidades, destacando los procesos civiles y penales. Al respecto, si bien la Mesa de Partes Electrónica del EJE facilita de sobremanera la virtualización del proceso en general y a las teleaudiencias en específico, no se debe supeditar el uso de estas últimas a la implementación del EJE, pues el contacto con el soporte material del proceso puede ser realizado mediante la lectura de escritos y piezas documentales digitalizadas, a las que accederán los magistrados mediante el empleo del Sistema Integrado Judicial (SIJ), y los abogados y litigantes harán lo propio por medio de

[3] Disponible en: https://justiciatv.pj.gob.pe/poder-judicial-implementa-plataforma-google-hangouts-meet-para-audiencias-virtuales-y-reuniones-administrativas/ 
notificaciones electrónicas, herramientas que el Poder Judicial peruano ya ha implementado a nivel nacional.

La Resolución Administrativa N. ${ }^{\circ} 000129$ 2020-CE-PJ, del 27 de abril de 2020, Protocolo denominado «Medidas de reactivación de los órganos jurisdiccionales y administrativos del Poder Judicial, posterior al levantamiento del aislamiento social obligatorio establecido por el D. S. N. ${ }^{\circ}$ 044-2020-PCM y prorrogado por los D. S. N. ${ }^{\circ}$ 051-2020-PCM y 064-2020PCM», texto normativo que en su acápite 5.8. prevé que el servicio de justicia se reactivará con - entre otras medidas - «audiencias online» y audiencias presenciales excepcionales, recibiendo las teleaudiencias reglamenteación transitoria con el «Protocolo Temporal para Audiencias Judiciales Durante el Periodo de Emergencia Sanitaria». Con ello se invierten los roles, las «audiencias online» - teleaudiencias - pasan a tener carácter de regla frente a las audiencias presenciales - con presencia física-como excepción.

Pese a los logros obtenidos con las teleaudiencias, resalta la ausencia de implementación de herramientas tecnológicas propias que las operativicen. Así mismo, en las teleaudiencias ya realizadas se advierten irregularidades, especialmente sobre los principios de inmediación y publicidad. Con el primero, se vulnera el principio al permitir la participación de los sujetos procesales -incluidos magistrados- mediante audio y no video, así como su salida y reingreso de la teleaudiencia durante su desarrollo. Con el segundo. se sacrifica el principio dado que para cualquier individuo ajeno al proceso le es imposible acceder a las audiencias «públicas», existiendo además desconocimiento de su programación.

Recuérdese que sobre este último punto el Tribunal Constitucional peruano señaló en el Exp. N. ${ }^{\circ}$ 02738-2014-PHC/TC que las audiencias llevadas por videoconferencia son constitucionales en la medida asegure la comunicación bidireccional en imagen y sonido —ambos_- y permitan el acceso de cualquier individuo a la sala de audiencias, sala que ahora se ha transformado en un espacio virtual inaccesible a terceros.

Se planea continuar utilizando la aplicación ya mencionada, cuanto menos, en lo que dure la emergencia sanitaria; no obstante, al utilizarse la plataforma de Google, la audiencia está sujeta a reglas definidas por empresas ajenas al órgano jurisdiccional que no fueron pensadas para audiencias judiciales, así como a peligros que pueden frustrarla como el boombig $^{[4]}$ o la caída de la red. Razón por la cual, si bien es de amparo exigir a la administración de justicia cubrir los niveles de publicidad requeridos en las audiencias presenciales, este principio debe ser relativizado (dicha situación es admisible bajo el contexto del aislamiento, pero será una exigencia a resolver para el futuro).

En otras realidades no existió la situación judicial de emergencia, siendo este problema prácticamente imperceptible para órganos jurisdiccionales, abogados y litigantes en cortes que cuentan procesos judiciales completamente virtualizados como es el caso del Tribunal Internacional de Catar (The Peninsula, 2020), a su vez aquellas con experiencia en audiencias virtuales —virtualización parcialsupieron afrontar mejor la emergencia.

En el Perú conviene implementar a nivel legislativo el modelo semiabierto de las teleaudiencias, orientado a criterios de oportunidad que hagan más permisivo su uso, pues aún no se ha alcanzado desaparecer la brecha tecnológica existente, y utilizar las TICs en el proceso «(...) no debería ocurrir por la fuerza, sino que debe ingresar como una opción más para que los usuarios del sistema judicial puedan asimilar, progresivamente, tanto sus ventajas como sus desventajas y los riesgos que esta tecnología trae consigo» (Amoni, 2013, p. 70).

[4] Interrupciones en una videoconferencia sin el permiso del anfitrión 
Todo ello con el fin de mejorar el servicio de justicia y prever situaciones de paralización como las causadas por el aislamiento social. Para ello, es necesario que se implemente una plataforma adecuada a las necesidades de una audiencia accesible para los sujetos procesales - y para quienes no lo son-, atendiendo a las particularidades de cada especialidad y a la naturaleza, forma y dinámica de los actos procesales, permitiendo en todo momento la grabación en audio y video de las teleaudiencias y su suspensión para determinados actos procesales —como aquellos que requieren mayor atención podemos citar a las conciliaciones, firma de actas, exámenes de testigos y peritos, entrevistas a menores, debates periciales, careos, objeciones, ofrecimiento de piezas documentales en audiencia, exhibición de pruebas materiales y audiencias complementarias-.

\section{CONCLUSIONES}

Las teleaudiencias mantienen una regulación tímida, pero se están haciendo presentes cada vez en más lugares y con mayor alcance, por lo que es necesario que las autoridades repiensen este instrumento como un vehículo para mejorar el servicio de administración de justicia.

Bajo el amparo de una suerte de peligro abstracto de vulneración de principios se pretende restringir al máximo la realización de teleaudiencias. Las teleaudiencias son equiparables a las audiencias presenciales, existiendo igual trato a elementos objetivos y subjetivos del proceso.

La reducción material de inmediación no es significativa como para sustentar la amplia restricción para su uso, el resto de principios procesales se verían potenciados; por lo que, no se puede considerar que existan fundamentos de naturaleza jurídica que sean válidos y suficientes para imponer un modelo cerrado.

Siempre que las reglas de las teleaudiencias sean claras y controladas por los actores que intervienen en la administración de justicia, los principios están plenamente garantizados.
Por consideraciones de oportunidad, es necesario una regulación prudente, para una futura reforma sobre las teleaudiencias, sujeta al modelo semiabierto, atendiendo a la brecha tecnológica del momento.

\section{REFERENCIAS}

Aba Catoira, A. (2009). La Tecnologización de la Prueba en el Proceso Penal, La Viedeoconferencia: objeciones y ventajas. AFDUDC, 9-37.

Albornoz Barrientos, J., \& Magdic, M. (2013). Marco jurídico de la utilización de videoconferencia en materia penal. Revista Chilena de Derecho y Tecnología, 229-260.

Amoni Reverón, G. A. (2013). El uso de la videoconferencia en cumplimiento del principio de inmediación. Revista del Instituto de Ciecias Jurídicas de Puebla, 67-85.

Amoni Reverón, G. A. (2014). Límites constitucionales a la audiencia telemática en el proceso penal Venezolano. Revista de Derecho, Comunicaciones y Nuevas Tecnologías.

Amrani-Mekki, S. (2008). El Impacto de las Nuevas Tecnologías sobre la Forma del Proceso. https://www.uv.es/coloquio/coloquio/ponen cias/6tecmek.pdf

Arellano, J., Cora, L., García, C., \& Sucunza, M. (2020). Estado de la Justicia en América Latina bajo el COVID-19. Medidas Generales adoptadas y uso de TICs en procesos judiciales. Santiago de Chile: Centro de Estudios de Justicia de las Américas (CEJA). https://cejamericas.org/2020/05/08/nuevapublicacion-reporte-ceja-estado-de-la-justicia-en-america-latina-bajo-el-covid-19/

Arnaiz Serrano, A. (10 de octubre de 2016). La experiencia Española en el Uso de Videoconferencia en el Proceso Penal. http://www. cienciaspenales.net/files/2016/10/8amayaarnaiz.pdf

Baral, I., \& Reissier, E. (Septembre de 2014). Vidéoconférence et principes fondamentaux du procès: publicité et inmediación 
en Espagne. http://ihej.org/wp-content/ uploads/2016/03/D\%C3\%A9cryptage-CasEspagnol.pdf

Binet, S. (17 de junio de 2005). L'utilisation des nouvelles technologies dans le procès civil: Vers une procédure civile intégralement informatisée? https://www.memoireonline. com/12/05/31/utilisation-nouvelles-technologies-proces-civil.html

Carrizo González-Castell, A. (2008). Las Nuevas Tecnologías y su incorporación al proceso penal. Pensamiento Jurídico, 129-152.

Carrizo González-Castell, A. (s/f). El Respeto de la Oralidad y los Principios Procesales en la Utilización de la Videoconferencia para la obtención de Pruebas en Materia Civil en la Unión Europea. Oralidad y Escritura en un Proceso Civil Eficiente, 175-186.

Consilium. (2013). Guía sobre videoconferencias en procedimientos judiciales transfonterizos. Bruselas.

CSJ R.N. N. ${ }^{\circ}$ 999-2016-Loreto, CSJ R.N. N. ${ }^{\circ}$ 999-2016-Loreto (Corte Suprema de Justicia del Perú 20 de Junio de 2017).

De Paula Ramos, V. (2019). La Prueba Testifical. Del subjetivismo al objetivismo, del aislamiento científico al diálogo con la psicología y la epistemología. (L. Criado Sánchez, Trad.) Madrid, San Sotero, España: Marcial Pons.

Dimeglio, A. (08 de enero de 2008). La visioconférence s'invite dans les procès. https://www. journaldunet.com/ebusiness/expert/21130/ la-visioconference-s-invite-dans-les-proces. shtml

Exp. N. ${ }^{\circ}$ 02738-2014-PHC, Exp. N. ${ }^{\circ}$ 02738-2014PHC (Tribunal Constitucional del Perú 30 de julio de 2015).

Fons Rodríguez, C. (2008). La Videoconferencia en el Proceso Civil (La telepresencia Judicial). Oralidad y escritura en un proceso civil eficiente [Coloquio de la Asociación Internacional de Derecho Procesal, 2008], 53-60.
Jeuland, E. (2007). Nouvelles technologies et procès civil, Rapport général pour les pays de droit civil. World Congress of Procedural Law (págs. 152-185). Bahía: Forense.

Laure, M. (2011). Visioconférence et droit à un procès équitable. http://www.revuedlf.com/ cedh/visioconference-et-droit-a-un-procesequitable/

Lillo Lobos, R. (2011). El Uso de Nuevas Tecnologías en el Sistema Judicial: experiencias y precauciones. Buenas Prácticas para la Implementación de Soluciones Tecnológicas en la Administración de Justicia, 117-140.

Marquina, J. (19 de mayo de 2020). https://lpderecho.pe/audiencia-judicial-virtual-existenobstaculos-peligro-inconcurrencia-conclusion-proceso/

Monroy Gálvez, J. (1993). Los Principios Procesales en el Código Procesal Civil de 1992. Themis, 35-48.

Monroy Gálvez, J. (1996). Introducción al Proceso Civil. Lima: Temis.

Montesinos García, A. (2009). La videoconferencia como instrumento probatorio en el proceso penal. Barcelona: Marcial Pons.

Ortells Ramos, M. (2002). Nuevas tecnologías y proceso jurisdiccional en el ámbito iberoamericano. Prueba, medidas cautelares y comunicaciones procesales. 221-255.

Pagés Lloveras, R. M. (2012). El Dilema entre la Oralidad y la Escritura en la Hora Actual. La Informática Judicial como una Tercera Vía. Derecho y Sociedad, 316-325.

Peláez Jiménez, D. F. (2015). EI Uso de las TICS Videoconferencia en la Audiencia de Juzgamiento del Procesado. Loja: Universidad de Loja.

Retamales Muñoz, R. A. (2017). Herramientas tecnológicas en la prueba testimonial en la reforma procesal civil. Santiago de Chile: Universidad de Chile.

Ríos Ruíz, A. (2016). La Justicia Electrónica en México: Visión Comparada con América 
Latina. Revista de la Facultad de Derecho de México, 389-422.

Tayro Tayro, E. A. (2016). La Videoconferencia en un Nuevo Enfoque del Principio de Inmediación Procesal. Revista Oficial del Poder Judicial, 547-559.

The Peninsula. (20 de abril de 2020). thepeninsulaqatar. https://thepeninsulaqatar.com/ article/09/04/2020/Qatar-International-Courtholds-first-fully-remote-online-hearing

Tirado Estrada, J. J. (2017). Videoconferencia, Cooperación Judicial Internacional y Debido Proceso. Rev. secr. Trib(10), 153-173. doi:10.16890/rstpr.a5.n10.p153

Torres López, E. (2009). Informática y Justicia en el Perú. Revista Oficial del Poder Judicial (05), 155-161.

\section{REFERENCIAS JURÍDICAS}

Código Procesal Penal (2004).

Convención de las Naciones Unidas contra la Corrupción (31 de octubre del 2003). https:// www.unodc.org/documents/mexicoandcentralamerica/publications/Corrupcion/ Convencion_de_las_NU_contra_la_Corrupcion.pdf

Convención de las Naciones Unidas contra la Delincuencia Organizada Transnacional y sus protocolos (15 de diciembre del 2000). https://www.unodc.org/documents/treaties/ UNTOC/Publications/TOC\%20Convention/ TOCebook-s.pdf

Convenio Europeo Relativo a la Asistencia Judicial en Materia Penal (20 de abril de 1959). https://www.pj.gob.pe/wps/wcm/connect/ 9e6b840049545a918a75fbcc4f0b1cf5/ Convenio + Europeo+de+Asistencia + Mutua +en + materia + penal.pdf?MO$\mathrm{D}=\mathrm{AJPERES}$

Convenio Iberoamericano sobre el Uso de la Videoconferencia en la Cooperación Internacional entre Sistemas de Justicia (3 de diciembre del 2010). http://www.comjib.
org/wp-content/uploads/imgDrupal/Convenio-Videoconferencia-ES-publicaciones_1.pdf

Decreto Supremo que declara en Emergencia Sanitaria a nivel nacional por el plazo de noventa (90) días calendario y dicta medidas de prevención y control del COVID-19. D. S. N. ${ }^{\circ}$ 008-2020-SA (11 de marzo de 2020). https://busquedas.elperuano.pe/normaslegales/decreto-supremo-que-declara-en-emergencia-sanitaria-a-nivel-decreto-supremo-n-008-2020-sa-1863981-2/

Decreto Supremo que declara Estado de Emergencia Nacional por las graves circunstancias que afectan la vida de la Nación a consecuencia del brote del COVID-19. D. S. N. ${ }^{\circ}$ 044-2020-PCM. (15 de marzo del 2020). https://busquedas.elperuano.pe/normaslegales/decreto-supremo-que-declara-estado-de-emergencia-nacional-po-decreto-supremo-n-044-2020-pcm-1864948-2/

Estatuto de la Corte Penal Internacional (17 de Julio de 1998). https://www.un.org/spanish/ law/icc/statute/spanish/rome_statute(s).pdf

Exp. N..$^{\circ}$ 02738-2014-PHC/TC (30 de julio del 2015). https://tc.gob.pe/jurisprudencia/2015/ 02738-2014-HC.pdf

Ley Orgánica 6/1985, de 1 de julio, del Poder Judicial. Madrid: Boletin Oficial del Estado. https://www.boe.es/buscar/pdf/1985/BOEA-1985-12666-consolidado.pdf

Lineamientos Para el Uso de la Videoconferencia en los Procesos Penales. Directiva 01-2014-CE-PJ. (07 de enero del 2014). https://www.pj.gob.pe/wps/wcm/connect/ aaae160043533423a27fa3694ab21750/ RA_004_2014_CE_PJ+-+07_01_2014.pdf? MOD $=$ AJPERES\&CACHEID $=$ aaae 1600435 33423a27fa3694ab21750

Medidas de reactivación de los órganos jurisdiccionales y administrativos del Poder Judicial, posterior al levantamiento del aislamiento social obligatorio establecido por el Decreto Supremo N. ${ }^{\circ}$ 044-2020-PCM y prorrogado por los Decretos Supremos 
N. os 051 y 064-2020-PCM. Resolución Administrativa N. ${ }^{\circ}$ 000129-2020-CE-PJ. (27 de abril del 2020). https://www.pj.gob.pe/wps/ wcm/connect/c08fc9004e16a5d3b949bb7ef3caaf63/RESOLUCION+ADMINISTRATIVA000129-2020-CE+\%2Banexo.pdf?MOD=AJ PERES\&CACHEID $=\mathrm{c} 08 \mathrm{fc} 9004 \mathrm{e} 16 \mathrm{a} 5 \mathrm{~d} 3 \mathrm{~b} 94$ 9bb7ef3caaf63

Plan de Prevención del Coronavirus (COVID-19) en el Poder Judicial. Resolución Administrativa 103-2020-CE-PJ. (11 de marzo del 2020). https://busquedas.elperuano.pe/down load/url/aprueban-el-plan-de-prevenciondel-coronavirus-covid-19-e-resolucion-administrativa-no-103-2020-ce-pj-1864256-1

Procedimiento Para el Uso de Equipos de Videoconferencia por Personal de los Órganos Jurisdiccionales y Administrativos del Poder Judicial Peruano. Directiva N. ${ }^{\circ}$ 005-2015-CE.-PJ. (15 de julio del 2015). https://www.pj.gob.pe/wps/wcm/connec $\mathrm{t} / 1 \mathrm{fb} 8 \mathrm{a} 88049 \mathrm{~cd} 6 \mathrm{cddbf70ffbb1314aca0/}$ RA_233_2015_CE_PJ+-+15_07_2015.pdf?
$\mathrm{MOD}=\mathrm{AJPERES} \& \mathrm{CACHEID}=1 \mathrm{fb} 8 \mathrm{a} 88049 \mathrm{c}$ d6cddbf70ffbb1314aca0

Protocolo Temporal para Audiencias Judiciales Virtuales durante el Período de Emergencia Sanitaria. Resolución Administrativa N. ${ }^{\circ}$ 000173-2020-CE-PJ (25 de junio de 2020). https://www.pj.gob.pe/wps/wcm/connect/ cf69f7804ec9465191b3f1cbea455c49/ RESOLUCION+ADMINISTRATIVA-0001732020-CE.pdf?MOD=AJPERES\&CACHEID= cf69f7804ec9465191b3f1cbea455c49

Proyecto de Reforma del Código Procesal Civil. Resolución Ministerial N0070-2018-JUS. (05 de marzo de 2018). https://es.scribd. com/document/375362203/proyecto-reforma-Codigo-Procesal-Civil\#from_embed

R.N.N. 9999-2016-Loreto(20dejuniode2017). https:// es.scribd.com/document/373766167/R-N999-2016-Loreto

Zivilprozessordnung [Código Procesal Civil Alemán] (2005). https://www.gesetze-im-internet.de/zpo/__128a.html 